\title{
HEAT TRANSEFER OF A DUSTY FLUID OVER A STRETCHING SHEET WITH INTERNAL HEAT GENERATION / ABSORPTION
}

\author{
A.K.Rauta ${ }^{1}$, S.K.Mishra ${ }^{2}$ \\ ${ }^{1}$ Lecturer, Department of Mathematics, S.K.C.G.College, Paralakhemundi, Odisha, India \\ ${ }^{2}$ Adjunct Professor, Centre for Fluid Dynamics Research, CUTM, Paralakhemundi, Odisha, India
}

\begin{abstract}
This paper pertains to investigate the heat transfer characteristics of two dimensional two phase flow in a porous medium over a stretching sheet with internal heat generation. The novelty of the present study is to consider the permeability parameter, space and temperature dependent internal heat generation along with various parameters like Froud number , heat source/sink parameter, Grashof number, Prandtl number, Eckert number, Volume fraction, fluid interaction parameter etc. The method of solution involves similarity transformation which reduces the partial differential equations into non linear ordinary differential equations. These non linear ordinary differential equations have been solved by applying Runge-Kutta 4-th order method with help of shooting technique. The temperature profiles for different values of flow parameters are presented in figures. It is observed from all the figures that the boundary conditions are satisfied asymptotically in all the cases which supporting the accuracy of the numerical results. All the figures shows that increasing values of any parameter increase the thermal boundary layer except the prandtl number and permeability parameter. AMS classification 76T10, $76 T 15$
\end{abstract}

Keywords: Heat source/sink, Volume fraction, Fluid - particle interaction parameter, Dusty fluid, Boundary layer flow, Stretching sheet, Eckert number, Grashof number, Prandtle number, Shooting techniques, Froud number, permeability parameter.

\section{INTRODUCTION}

The study of heat source / sink effects on heat transfer of dusty fluid flows over a continuously moving solid surface has many important applications including boundary layer flow over heat treated material between feed roll and a windup roll, rolling and manufacturing of plastic films ,cooling of an infinite metallic plate in cooling bath, the boundary layer along a liquid film in condensation process, and aerodynamic extrusion of plastic sheets etc . The momentum and Heat transfer over a stretching sheet have been studied because of its ever increasing usage in polymer processing industry, manufacturing of artificial fibers, viscoelastic fluid flow and in some application of dilute polymer solution, such as the $5.4 \%$ solution of polyisobutylene in cetane, curing of plastics, manufacture of printed circuitry, pulp-insulated cables etc .

Sakiadis B.C.[20] was the pioneer of studying the boundary layer flow over a stretched surface moving with a constant velocity in 1961.Then many researchers extended the above study with the effect of Heat Transfer . Grubka et.al[6] investigated the temperature field in the flow over a stretching surface when subject to uniform heat flux .Anderson [9] discussed a new similarity solution for the temperature fields. Sharidan[21] presented similarity solutions for unsteady boundary layer flow and heat Transfer due to stretching sheet . Chen [5] investigated mixed convection of a power law fluid past a stretching surface in presence of thermal radiation and magnetic field. Chakrabarti et.al[[11] have studied the hydro magnetic flow and Heat Transfer over a stretching sheet .Crane [12] has obtained the Exponential solution for planar viscous flow of linear stretching sheet.

The problem of two phase suspension flow is solved in the frame work of a model of a two-way coupling model or a two-fluid approach. The heat transfer over a stretching porous sheet subjected to power law heat flux in presence of heat source has been considered by Hitesh Kumar[9]. Mukhopadhyaya[24] recently has studied the heat transfer analysis of unsteady flow of Maxwell fluid over a stretching sheet in presence of heat source/sink. Singh and Singh et.al [(22] have studied MHD flow with viscous dissipation and chemical reaction over a stretching porous plate in porous medium. Robert A.Van Gorder et.al.[18] have studied the convective heat transfer in a conducting fluid over a permeable stretching surface with suction and internal heat generation/absorption. Kannan et al.[10] studied the free convection in an infinite porous dusty medium induced by pulsating point heat source. B.J. Gireesha et.al[4] have studied the effect of hydrodynamic laminar boundary layer flow and heat Transfer of a dusty fluid over an unsteady stretching surface in presence of non uniform heat source/sink .They have examined the Heat Transfer characteristics for two type of boundary conditions namely variable wall temperature and variable Heat flux. R.K.Ramesh et.al [16] have investigated the momentum and heat transfer characteristics in hydrodynamic flow of dusty fluid over an inclined stretching sheet with non uniform heat source/sink .B.G. Gireesh et.al [3] also studied the mixed 
convective flow a dusty fluid over a stretching sheet in presence of thermal radiation, space dependent heat source/sink. Manjunatha[14] et al recently have studied the heat transfer in MHD flow of fluid-particle suspension over an impermeable surface through a porous medium with non uniform heat source /sink.

The objective of the present analysis is to consider the mass and heat transfer aspect of the works of two phase flow in a porous medium over a stretching sheet with internal heat generation and absorption. Here, the particles will be allowed to diffuse through the carrier fluid i.e. the random motion of the particles shall be taken into account because of the small size of the particles. This can be done by applying the kinetic theory of gases and hence the motion of the particles across the streamline due to the concentration and pressure diffusion. We have considered the terms related to the heat added to the system to slip-energy flux in the energy equation of particle phase. The momentum equation for particulate phase in normal direction, heat due to conduction and viscous dissipation in the energy equation of the particle phase have been considered for better understanding of the boundary layer characteristics. The effects of volume fraction on skin friction, heat transfer and other boundary layer characteristics also have been studied. The governing equation are reduced into system of ODE and solved by Shooting Techinique using Runge-Kutta Method.

\section{MATHEMATICAL FORMULATION AND SOLUTION:}

Consider a steady two dimensional laminar boundary layer of an incompressible viscous dusty fluid over a vertical stretching sheet .The flow is generated by the action of two equal and opposite forces along the $\mathrm{x}$-axis and $\mathrm{y}$-axis being normal to the flow. The sheet being stretched with the velocity $U_{w}(x)$ along the $x$-axis, keeping the origin fixed in the fluid of ambient temperature T. Both the fluid and the dust particle clouds are suppose to be static at the beginning. The dust particles are assumed to be spherical in shape and uniform in size and number density of the dust particle is taken as a constant throughout the flow.

The governing equations of steady two dimensional boundary layer incompressible flows of dusty fluids are given by

$$
\begin{gathered}
\frac{\partial}{\partial x} \vec{F}\left(u_{f}\right)+\frac{\partial}{\partial y} \vec{G}\left(u_{f}\right)+H\left(u_{f}\right)=S\left(u_{f}, u_{p}, T, T_{p}\right) \\
\frac{\partial}{\partial x} \vec{F}\left(u_{p}\right)+\frac{\partial}{\partial y} \vec{G}\left(u_{p}\right)+H\left(u_{p}\right)=S_{p}\left(u_{f}, u_{p}, T, T_{p}\right)
\end{gathered}
$$

Where $H\left(u_{f}\right)=0, H\left(u_{p}\right)=0$

$$
\begin{gathered}
\vec{F}\left(u_{f}\right)=\left[\begin{array}{c}
u \\
(1-\varphi) \rho u^{2} \\
\rho c_{p} u T
\end{array}\right], \vec{G}\left(u_{f}\right)=\left[\begin{array}{c}
v \\
(1-\varphi) \rho u v \\
\rho c_{p} v T
\end{array}\right], \vec{F}\left(u_{p}\right)=\left[\begin{array}{c}
\rho_{p} u_{p} \\
\rho_{p} u_{p}{ }^{2} \\
\rho_{p} u_{p} v_{p} \\
\rho_{p} c_{s} u_{p} T_{p}
\end{array}\right], \vec{G}\left(u_{p}\right)=\left[\begin{array}{c}
\rho_{p} v_{p} \\
\rho_{p} u_{p} v_{p} \\
\rho_{p} v_{p}^{2} \\
\rho_{p} c_{s} v_{p} T_{p}
\end{array}\right] \\
S\left(u_{f}, u_{p}, T, T_{p}\right)=\left[\begin{array}{c}
\mu \frac{\partial^{2} u}{\partial y^{2}}-\frac{\rho_{p}}{\tau_{p}}\left(u-u_{p}\right)+g \beta^{*}\left(T-T_{\infty}\right)-\frac{\mu}{k_{p}} \frac{u}{\rho} \\
k(1-\varphi) \frac{\partial^{2} T}{\partial y^{2}}+\frac{\rho_{p} c_{s}}{\tau_{T}}\left(T_{p}-T\right)+\frac{\rho_{p}}{\tau_{p}}\left(u_{p}-u\right)^{2}+\mu(1-\varphi)\left(\frac{\partial u}{\partial y}\right)^{2}+\frac{q^{\prime \prime \prime}}{\rho c_{p}}
\end{array}\right] \\
S_{p}\left(u_{f}, u_{p}, T, T_{p}\right)=\left[\begin{array}{c}
\frac{\partial}{\partial y}\left(\varphi \mu_{s} \frac{\partial u_{p}}{\partial y}\right)+\frac{\rho_{p}}{\tau_{p}}\left(u-u_{p}\right)+\varphi\left(\rho_{s}-\rho\right) g \\
\frac{\partial}{\partial y}\left(\varphi \mu_{s} \frac{\partial v_{p}}{\partial y}\right)+\frac{\rho_{p}}{\tau_{p}}\left(v-v_{p}\right) \\
\frac{\partial y}{\partial y}\left(\varphi k_{s} \frac{\partial T_{p}}{\partial y}\right)-\frac{\rho_{p}}{\tau_{p}}\left(u-u_{p}\right)^{2}+\varphi \mu_{s}\left(u_{p} \frac{\partial^{2} u_{p}}{\partial y^{2}}+\left(\frac{\partial u_{p}}{\partial y}\right)^{2}\right)-\frac{\rho_{p} c_{s}}{\tau_{T}}\left(T_{p}-T\right)
\end{array}\right]
\end{gathered}
$$

With boundary conditions

$\left.\begin{array}{l}u=U_{w}(x)=c x, v=0 \text { at } y=0 \\ \rho_{p}=\omega \rho, u=0, u_{p}=0, v_{p} \rightarrow v \text { as } y \rightarrow \infty\end{array}\right\}$

Where $\omega$ is the density ratio in the main stream . 
Similarly the corresponding boundary condition for $\mathrm{T}$ and $T_{p}$ are given by

$\left.\begin{array}{l}T=T_{w}=T_{\infty}+A\left(\frac{x}{l}\right)^{2} \text { at } y=0 \\ T \rightarrow T_{\infty}, T_{p} \rightarrow T_{\infty} \text { as } y \rightarrow \infty\end{array}\right\}$

Where A is a positive constant $l=\sqrt{\frac{v}{c}}$ is a characteristic length.

For most of the gases $\tau_{p} \approx \tau_{T}$,

$k_{s}=k \frac{c_{S}}{c_{p}} \frac{\mu_{S}}{\mu}$ if $\frac{c_{S}}{c_{p}}=\frac{2}{3 P_{r}}$

Introducing the following non dimensional variables in equation (2.1) and (2.2)

$u=c x f^{\prime}(\eta), v=-\sqrt{c v} f(\eta), \eta=\sqrt{\frac{c}{v}} y$,

$u_{p}=c x F(\eta), v_{p}=\sqrt{c v} G(\eta), \rho_{r}=H(\eta)$

$\theta(\eta)=\frac{T-T_{\infty}}{T_{w}-T_{\infty}}, \theta_{p}(\eta)=\frac{T_{p}-T_{\infty}}{T_{w}-T_{\infty}}$,

Where $\mathrm{T}-\mathrm{T}_{\infty}=\mathrm{A}\left(\frac{\mathrm{x}}{\mathrm{l}}\right)^{2} \theta, \mathrm{T}_{\mathrm{p}}-\mathrm{T}_{\infty}=\mathrm{A}\left(\frac{\mathrm{x}}{\mathrm{l}}\right)^{2} \theta_{\mathrm{p}}$

$\beta=\frac{1}{c \tau_{p}}, \epsilon=\frac{v_{s}}{v}, P_{r}=\frac{\mu c_{p}}{k}, E_{c}=\frac{c^{2} l^{2}}{A c_{p}}=\frac{v c}{A c_{p}}$

$\mathrm{F}_{\mathrm{r}}=\frac{\mathrm{c}^{2} \mathrm{x}}{\mathrm{g}}, \gamma=\frac{\rho_{\mathrm{s}}}{\rho}, \mathrm{G}_{\mathrm{r}}=\frac{\mathrm{g} \beta^{*}\left(\mathrm{~T}-\mathrm{T}_{\infty}\right)}{\mathrm{c}^{2} \mathrm{x}}, \nu=\frac{\mu}{\rho}$

$\mathrm{S}=\frac{\mathrm{ck}}{v}, \mathrm{q}^{\prime \prime \prime}=\left(\frac{\mathrm{kU}(\mathrm{x})}{\mathrm{x} v}\right)\left[\mathrm{A}^{*}\left(\mathrm{~T}_{\mathrm{w}}-\mathrm{T}_{\infty}\right) \mathrm{f}^{\prime}(\eta)+\mathrm{B}^{*}\left(\mathrm{~T}-\mathrm{T}_{\infty}\right)\right]$

We get the following non dimensional form.

$$
\begin{aligned}
& H F+H G^{\prime}+G H^{\prime}=0 \\
& f^{\prime \prime \prime}(\eta)+f(\eta) f^{\prime \prime}(\eta)+\frac{1}{(1-\varphi)} \beta H(\eta)\left[F(\eta)-f^{\prime}(\eta)\right]+G r \theta-\frac{1}{s} f^{\prime}(\eta)=0 \\
& G(\eta) F^{\prime}(\eta)+[F(\eta)]^{2}-\epsilon F^{\prime \prime}(\eta)-\beta\left[f^{\prime}(\eta)-F(\eta)\right]-\frac{1}{F r}\left(1-\frac{1}{\gamma}\right)=0 \\
& G G^{\prime}-\epsilon G^{\prime \prime}+\beta[f+G]=0 \\
& \theta^{\prime \prime}-\operatorname{Pr}\left(2 f^{\prime} \theta-f \theta^{\prime}\right)+\frac{2}{3} \frac{\beta}{1-\varphi} H\left[\theta_{p}-\theta\right]+\frac{1}{1-\varphi} \operatorname{Pr} E_{c} \beta H\left[F-f^{\prime}\right]^{2}+\operatorname{Pr} E_{c} f^{\prime 2}+A^{*} f^{\prime}(\eta)+B^{*} \theta(\eta)=0 \\
& 2 F \theta_{p}+G \theta_{p}^{\prime}+\beta\left[\theta_{p}-\theta\right]-\frac{\epsilon}{P r} \theta_{p}^{\prime \prime}+\frac{3}{2} \beta E c \operatorname{Pr}\left[f^{\prime}-F\right]^{2}-\frac{3}{2} \epsilon \operatorname{EcPr}\left[F F^{\prime \prime}+\left(F^{\prime}\right)^{2}\right]=0
\end{aligned}
$$

with boundary conditions

$G^{\prime}(\eta)=0, f(\eta)=0, f^{\prime}(\eta)=1$,

$F^{\prime}(\eta)=0, \theta(\eta)=1, \theta_{p}^{\prime}=0$ as $\eta \rightarrow 0$

$f^{\prime}(\eta)=0, F(\eta)=0, G(\eta)=-f(\eta)$,

$H(\eta)=\omega, \theta(\eta) \rightarrow 0, \theta_{p}(\eta) \rightarrow 0$ as $\eta \rightarrow \infty$ 


\section{SOLUTION OF THE PROBLEM}

Here in this problem the value of $f^{\prime \prime}(0), F(0), G(0), H(0), \theta^{\prime}(0), \theta_{p}(0)$ are not known but $f^{\prime}(\infty)=0, F(\infty)=0, G(\infty)=-f(\infty), H(\infty)=$ $\omega, \theta(\infty)=0, \theta_{p}(\infty)=0$ are given. We use Shooting method to determine the value of $f^{\prime \prime}(0), F(0), G(0), H(0), \theta^{\prime}(0), \theta_{p}(0)$.We have supplied $f^{\prime \prime}(0)=\propto_{0}$ and $f^{\prime \prime}(0)=\propto_{1}$. The improved value of $f^{\prime \prime}(0)=\propto_{2}$ is determined by utilizing linear interpolation formula described in equations (A). Then the value of $f^{\prime}\left(\propto_{2}, \infty\right)$ is determined by using Runge-Kutta method. If $f^{\prime}\left(\propto_{2}, \infty\right)$ is equal to $f^{\prime}(\infty)$ up to a certain decimal accuracy, then $\alpha_{2}$ i.e $f^{\prime \prime}(0)$ is determined, otherwise the above procedure is repeated with $\propto_{0}=\propto_{1}$ and $\propto_{1}=\propto_{2}$ until a correct $\alpha_{2}$ is obtained. The same procedure described above is adopted to determine the correct values of $F(0), G(0), H(0), \theta^{\prime}(0), \theta_{p}(0)$.

The essence of Shooting technique to solve a boundary value problem is to convert the boundary value problem into initial value problem. In this problem the missing value of $\theta^{\prime}(0)$ and $f^{\prime \prime}(0)$ for different set of values of parameter are chosen on hit and trial basis such that the boundary condition at other end i.e. the boundary condition at infinity $\left(\eta_{\infty}\right)$ are satisfied.A study was conducting to examine the effect of step size as the appropriate values of step size $\Delta \eta$ was not known to compare the initial values of $\theta^{\prime}(0)$ and $f^{\prime \prime}(0)$.If they agreed to about 6 significant digits, the last value of $\eta_{\infty}$ used was considered the appropriate value; otherwise the procedure was repeated until further change in $\eta_{\infty}$ did not lead to any more change in the value of $\theta^{\prime}(0)$ and $f^{\prime \prime}(0)$. The step size $\Delta \eta=0.125$ has been found to ensure to be the satisfactory convergence criterion of $1 \times 10^{-6}$. The solution of the present problem is obtained by numerical computation after finding the infinite value for $\eta$. It has been observed from the numerical result that the approximation to $\theta^{\prime}(0)$ and $f^{\prime \prime}(0)$ are improved by increasing the infinite value of $\eta$ which is finally determined as $\eta=10.0$ with a step length of 0.125 beginning from $\eta=0$. Depending upon the initial guess and number of steps $\mathrm{N}$. the values of $f^{\prime \prime}(0)$ and $\theta^{\prime}(0)$ are obtained from numerical computations which are given in table -1 for different parameters.

\section{GRAPHICAL REPRESENTATION:}
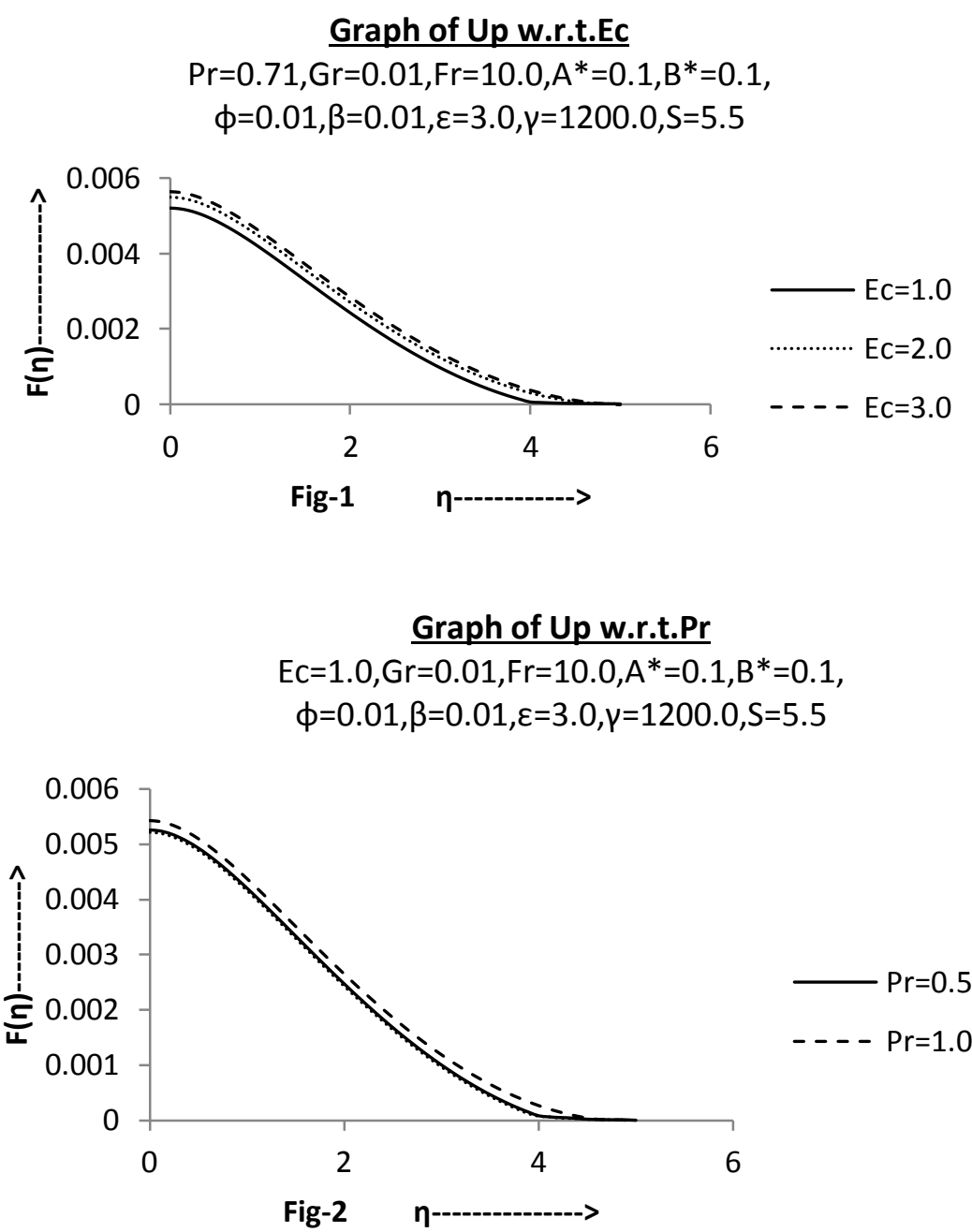

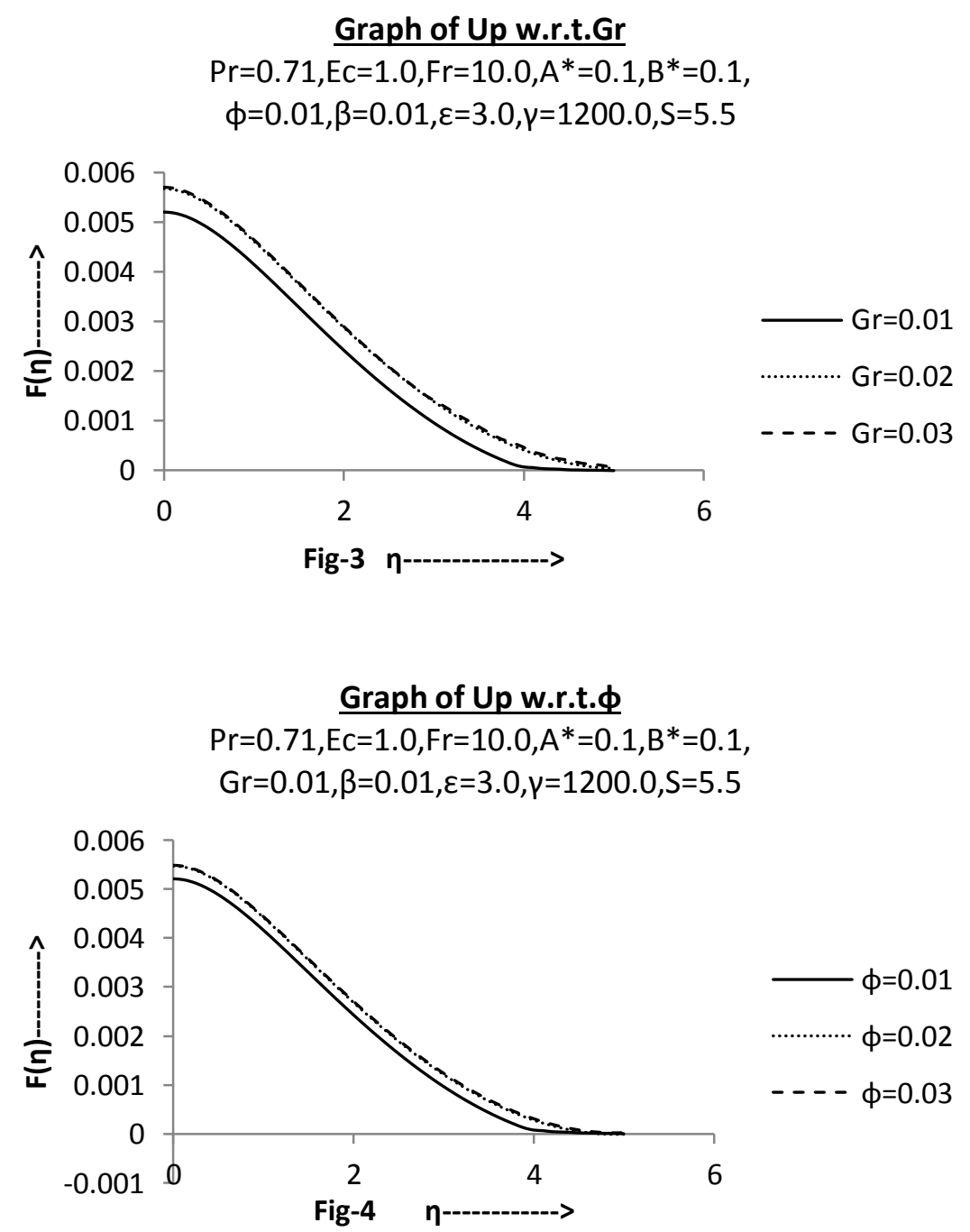

\section{Graph of Up w.r.t. $\beta$}

$\operatorname{Pr}=0.71, \mathrm{Ec}=1.0, \mathrm{Fr}=10.0, \mathrm{~A}^{*}=0.1, \mathrm{~B}^{*}=0.1$,

$\mathrm{Gr}=0.01, \phi=0.01, \varepsilon=3.0, \gamma=1200.0, \mathrm{~S}=5.5$

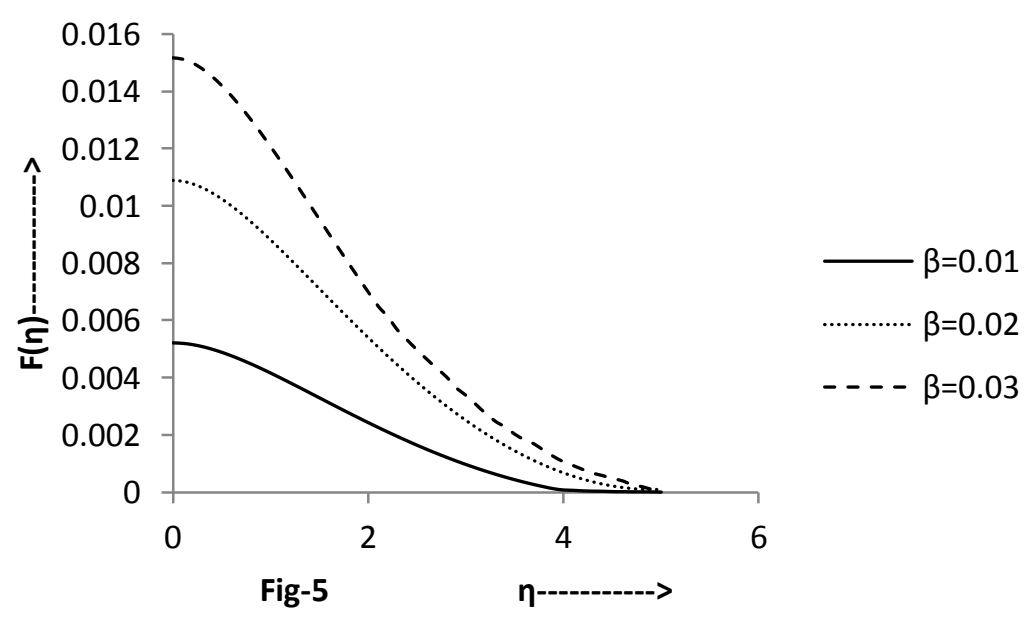




\section{Graph of Up w.r.t.S}

$\operatorname{Pr}=0.71, \mathrm{Ec}=1.0, \mathrm{Fr}=10.0, \mathrm{~A}^{*}=0.1, \mathrm{~B}^{*}=0.1$, $\mathrm{Gr}=0.01, \phi=0.01, \varepsilon=3.0, \gamma=1200.0, \beta=0.01$

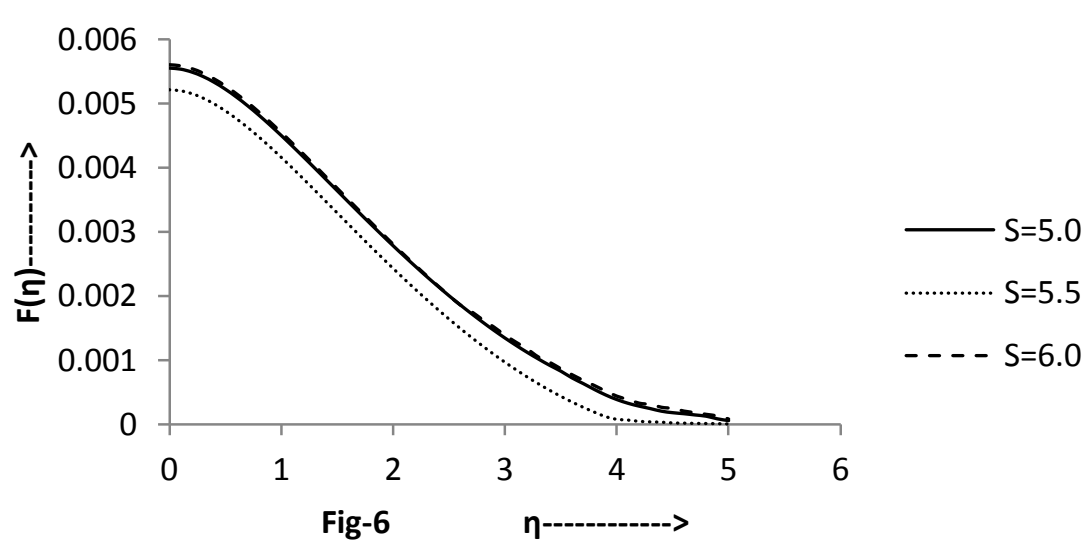

\section{Graph of Up w.r.t.A*}

$\operatorname{Pr}=0.71, \mathrm{Ec}=1.0, \mathrm{Fr}=10.0, \mathrm{~S}=5.5, \mathrm{~B}^{*}=0.1$, $\mathrm{Gr}=0.01, \phi=0.01, \varepsilon=3.0, \gamma=1200.0, \beta=0.01$

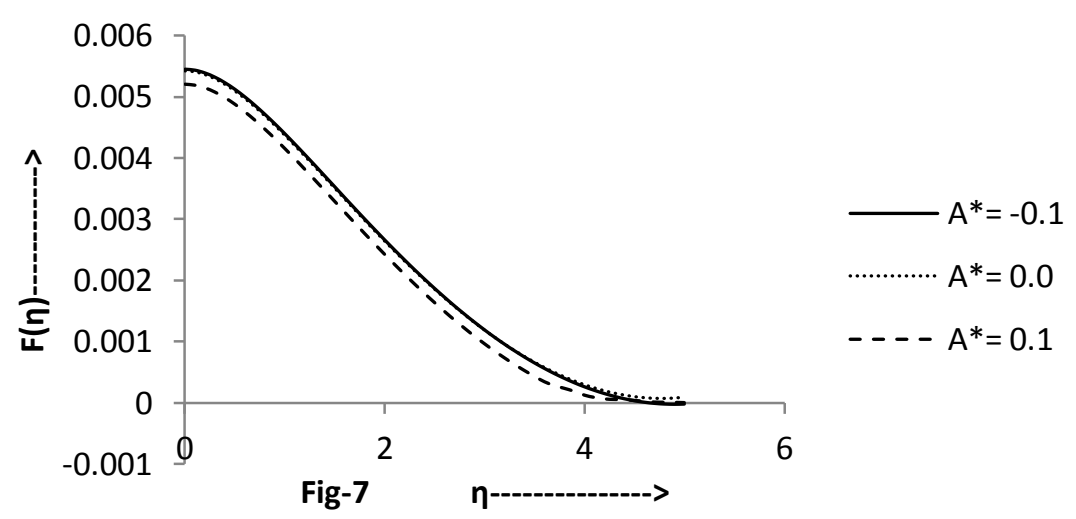

Graph of Up w.r.t.B*

$\operatorname{Pr}=0.71, \mathrm{Ec}=1.0, \mathrm{Fr}=10.0, \mathrm{~S}=5.5, \mathrm{~A}^{*}=0.1$, $\mathrm{Gr}=0.01, \phi=0.01, \varepsilon=3.0, \gamma=1200.0, \beta=0.01$

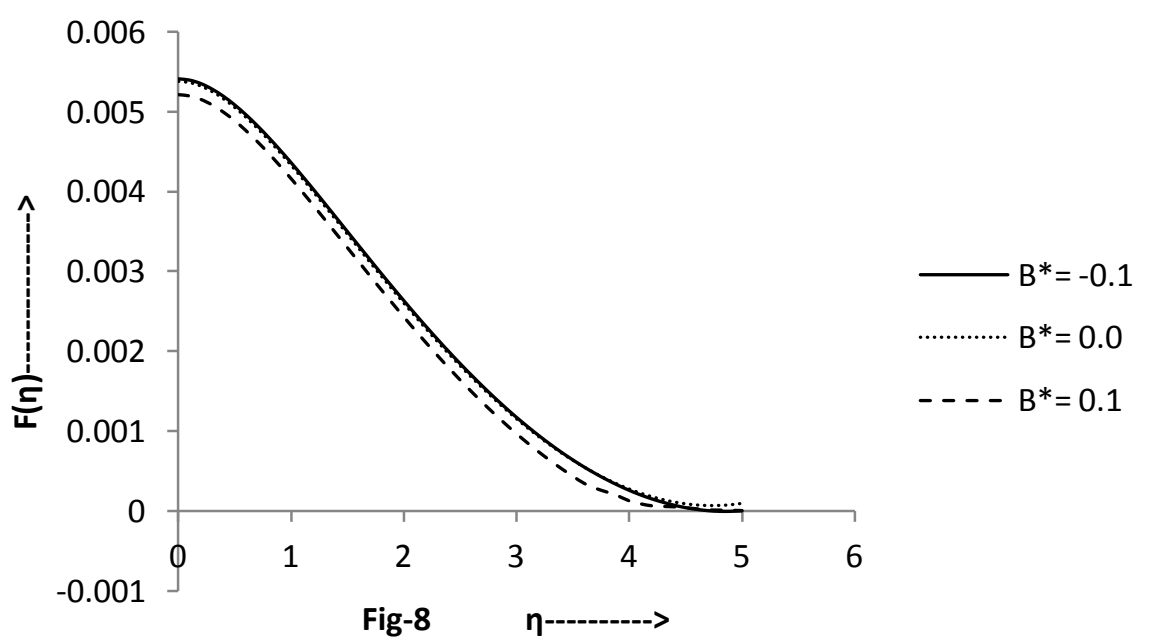




\section{Graph of $\theta$ w.r.t.Ec}

$\operatorname{Pr}=0.71, \mathrm{~B}^{*}=0.1, \mathrm{Fr}=10.0, \mathrm{~S}=5.5, \mathrm{~A}^{*}=0.1$, $\mathrm{Gr}=0.01, \phi=0.01, \varepsilon=3.0, \gamma=1200.0, \beta=0.01$

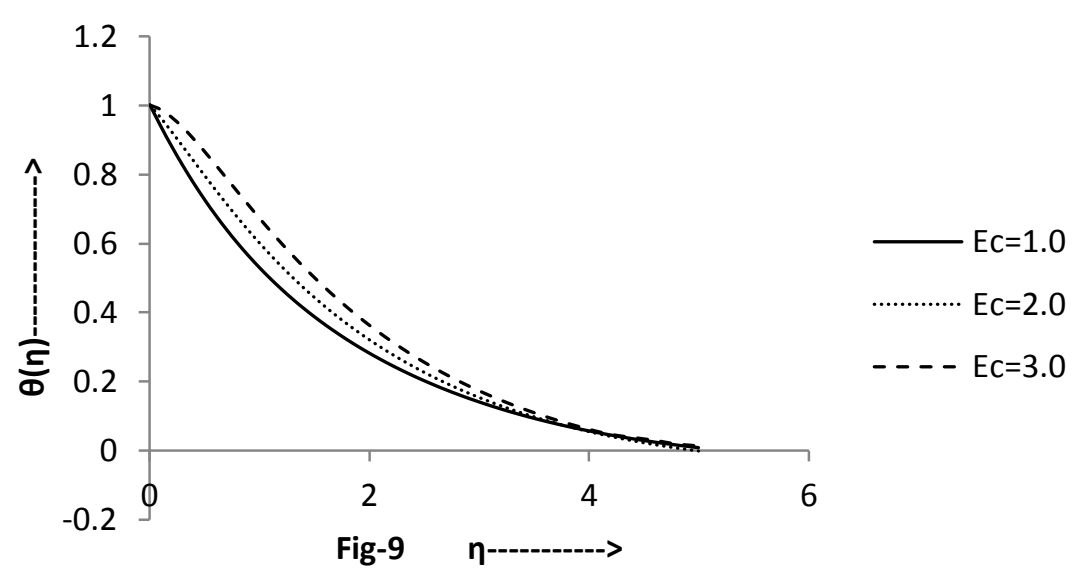

\section{Graph of $\theta$ w.r.t.Pr}

$E C=1.0, B^{*}=0.1, F r=10.0, S=5.5, A^{*}=0.1$, $\mathrm{Gr}=0.01, \phi=0.01, \varepsilon=3.0, \gamma=1200.0, \beta=0.01$
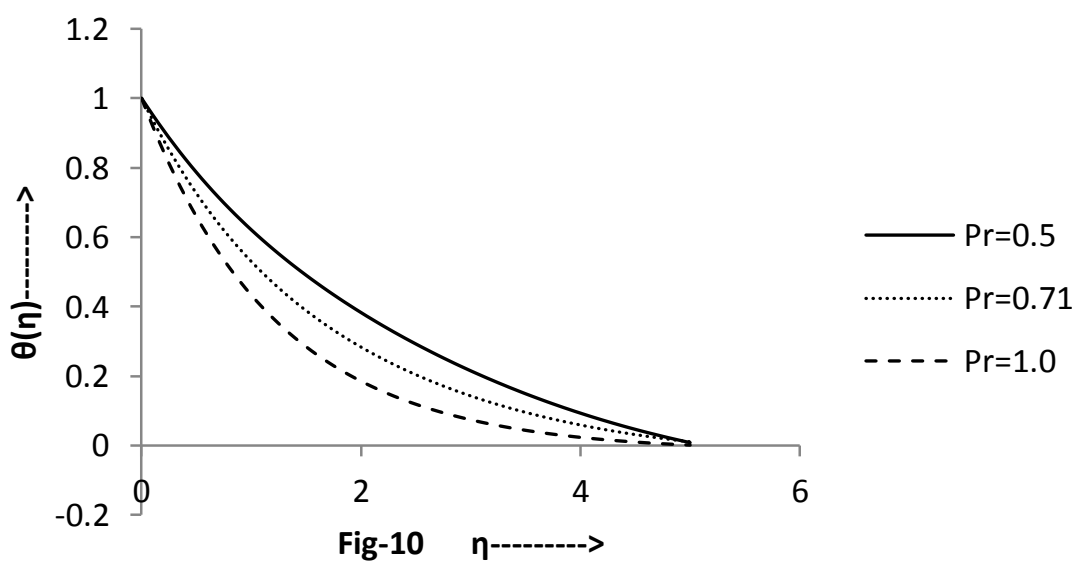

\section{Graph of $\theta$ w.r.t.A*}

$\mathrm{EC}=1.0, \mathrm{~B}^{*}=0.1, \mathrm{Fr}=10.0, \beta=0.01, \mathrm{~S}=5.5$, $\operatorname{Pr}=0.71, \mathrm{Gr}=0.01, \varepsilon=3.0, \gamma=1200.0, \phi=0$.
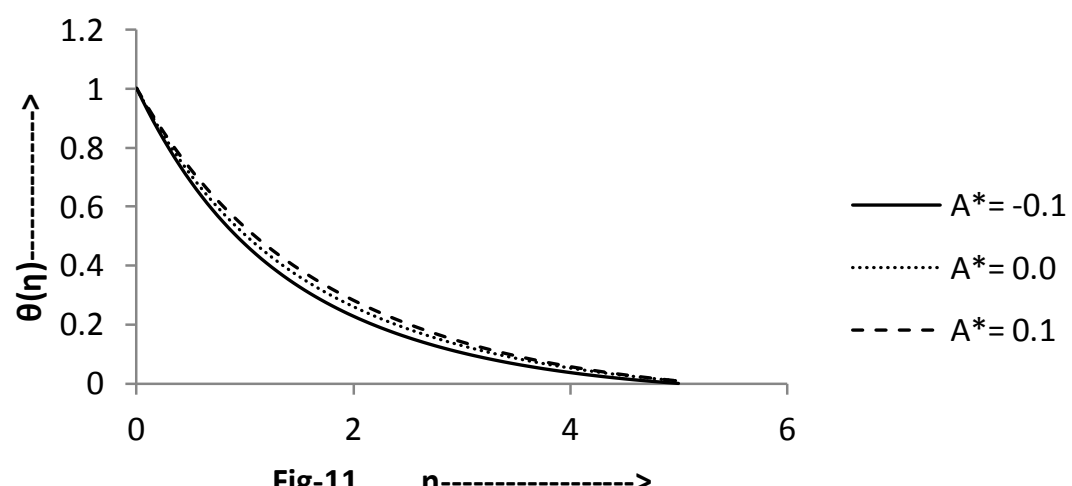

Fig-11 n----------------> 


\section{Graph of $\theta$ w.r.t.B*}

$\mathrm{EC}=1.0, \mathrm{~A}^{*}=0.1, \mathrm{Fr}=10.0, \beta=0.01, \mathrm{~S}=5.5$, $\operatorname{Pr}=0.71, \mathrm{Gr}=0.01, \varepsilon=3.0, \gamma=1200.0, \phi=0$.

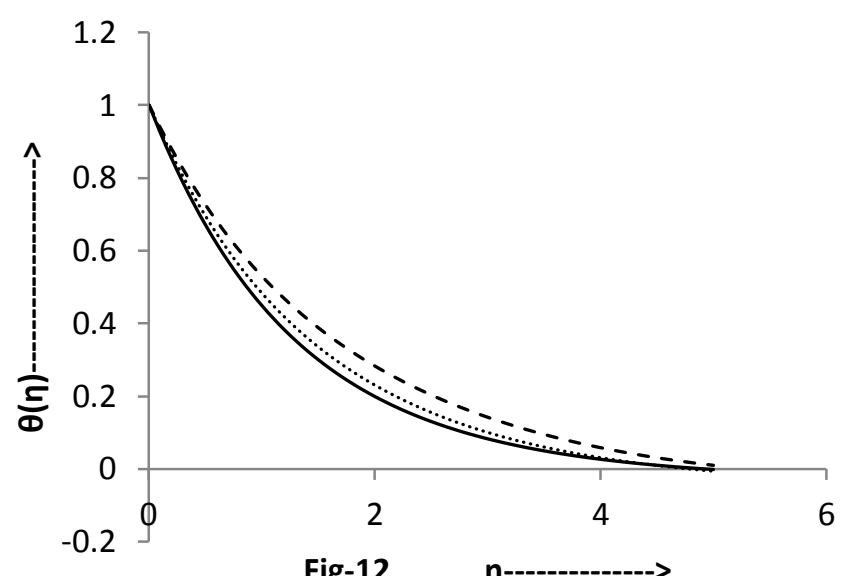

$$
\begin{aligned}
B^{*} & =-0.1 \\
\cdots \cdots \cdots . . B^{*} & =0.0 \\
---B^{*} & =0.1
\end{aligned}
$$

Fig-12 n------------>

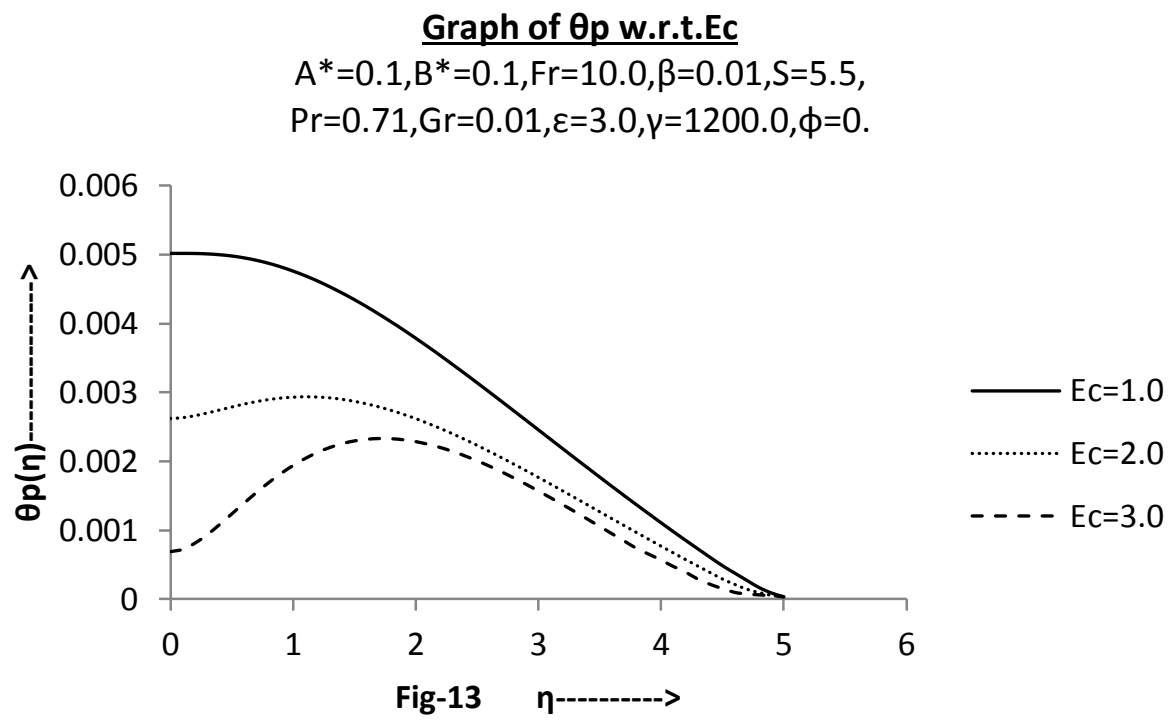

\section{Graph of $\theta p$ w.r.t.Pr}

$\mathrm{EC}=1.0, \mathrm{~A}^{*}=0.1, \mathrm{Fr}=10.0, \beta=0.01, \mathrm{~S}=5.5$, $B^{*}=0.1, G r=0.01, \varepsilon=3.0, \gamma=1200.0, \phi=0$.

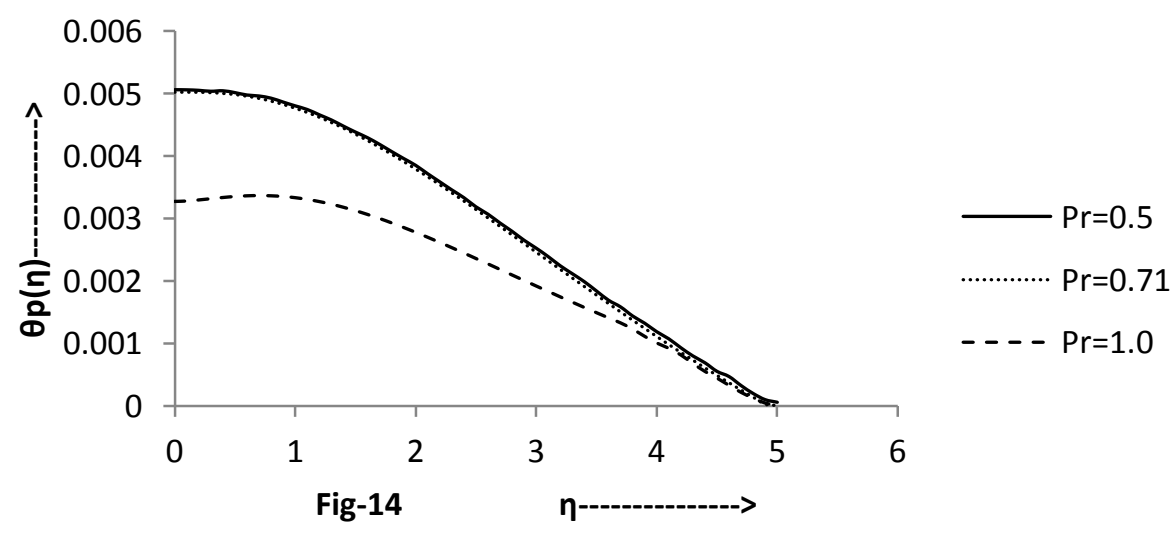




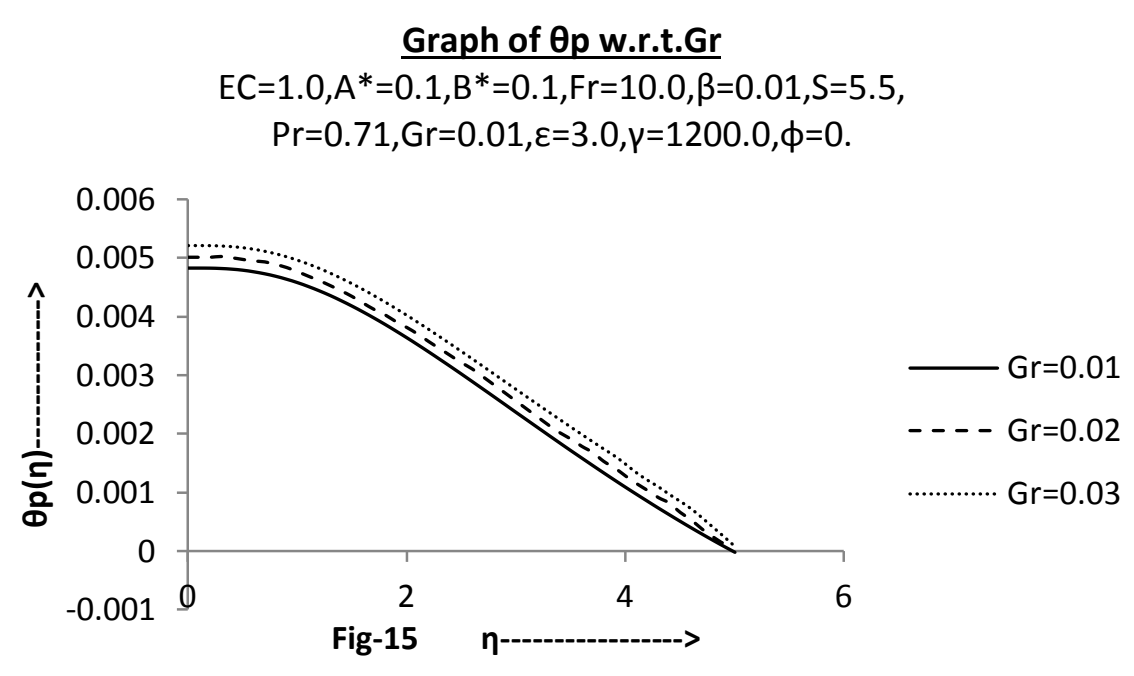

Graph of $\theta p$ w.r.t. $\phi$

$\mathrm{EC}=1.0, \mathrm{~A}^{*}=0.1, \mathrm{~B}^{*}=0.1, \mathrm{Fr}=10.0, \beta=0.01, \mathrm{~S}=5.5$,

$\operatorname{Pr}=0.71, \mathrm{Gr}=0.01, \varepsilon=3.0, \gamma=1200.0$

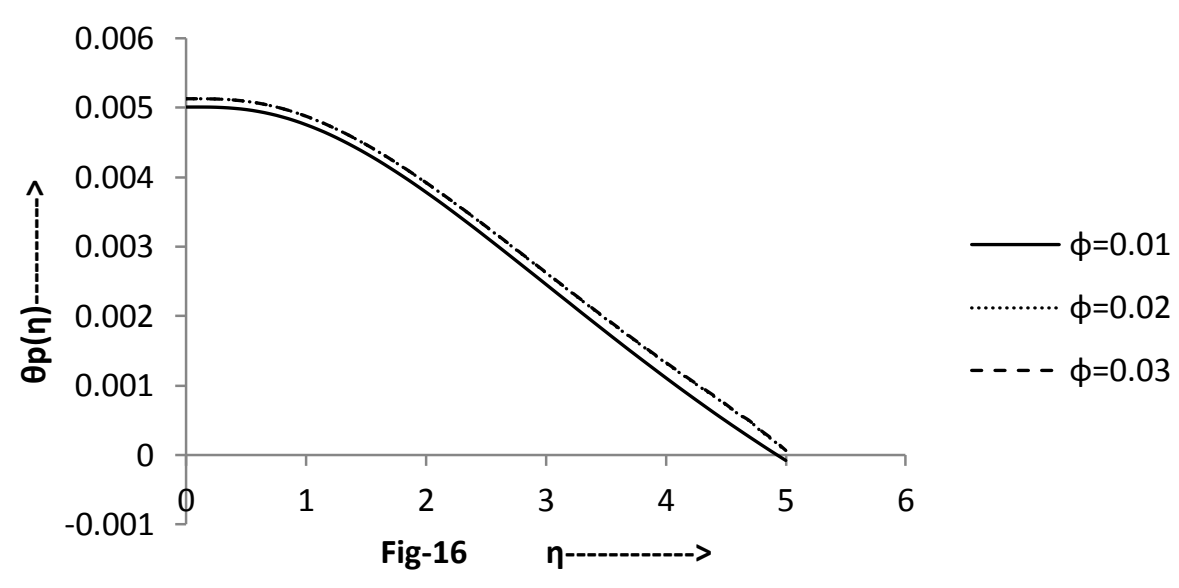

Graph of $\theta p$ w.r.t. $\beta$

$\mathrm{EC}=1.0, \mathrm{~A}^{*}=0.1, \mathrm{~B}^{*}=0.1, \mathrm{Fr}=10.0, \phi=0.01$,

$\mathrm{S}=5.5, \mathrm{Pr}=0.71, \mathrm{Gr}=0.01, \varepsilon=3.0, \gamma=1200.0$

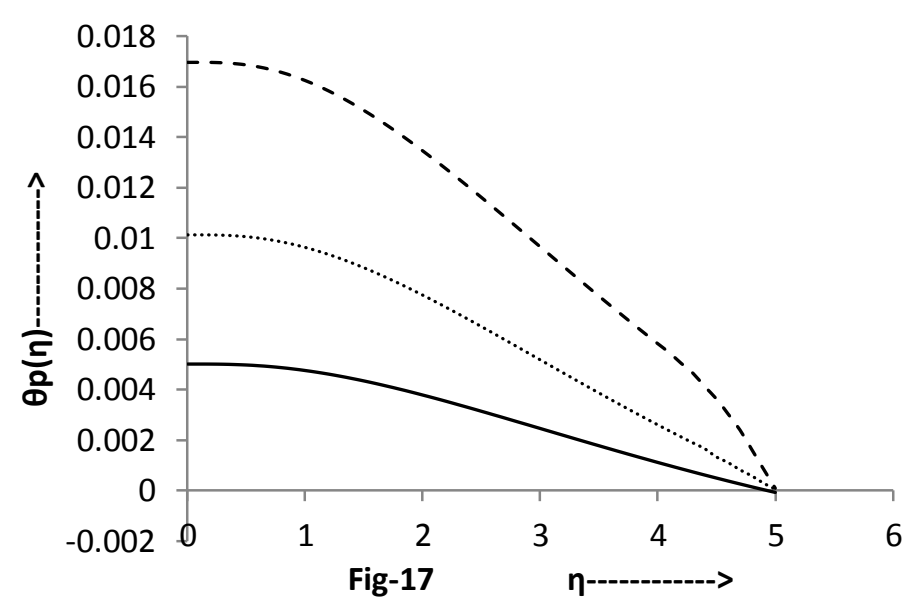

$\beta=0.01$

$\beta=0.02$

$---\beta=0.03$ 


\section{Graph of $\theta p$ w.r.t.S}

$\mathrm{EC}=1.0, \mathrm{~A}^{*}=0.1, \mathrm{~B}^{*}=0.1, \mathrm{Fr}=10.0, \beta=0.01$, $\phi=0.01, \operatorname{Pr}=0.71, \mathrm{Gr}=0.01, \varepsilon=3.0, \gamma=1200.0$

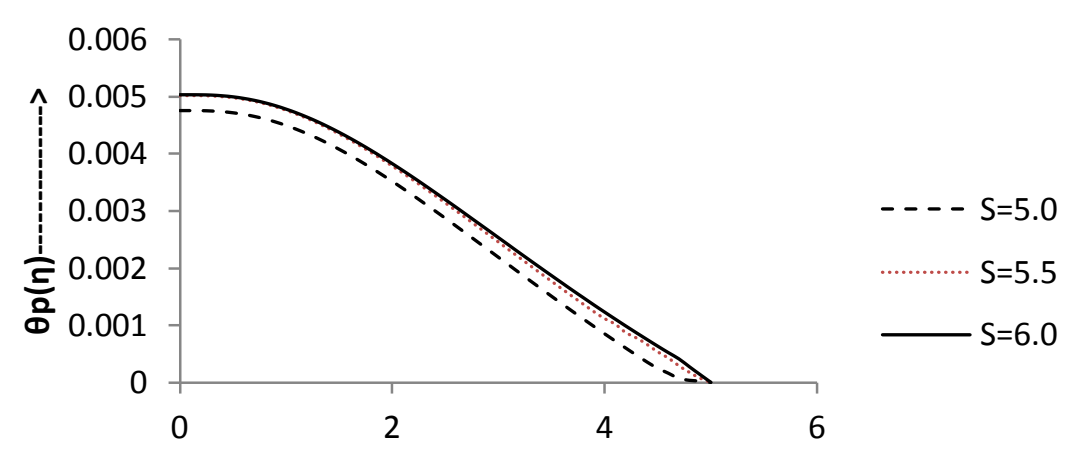

Fig-18 n------------>

\section{Graph of $\theta$ p w.r.t.A*}

$\mathrm{EC}=1.0, \mathrm{~B}^{*}=0.1, \mathrm{Fr}=10.0, \beta=0.01, \phi=0.01$

$\operatorname{Pr}=0.71, \mathrm{Gr}=0.01, \varepsilon=3.0, \gamma=1200.0, \mathrm{~S}=5.5$

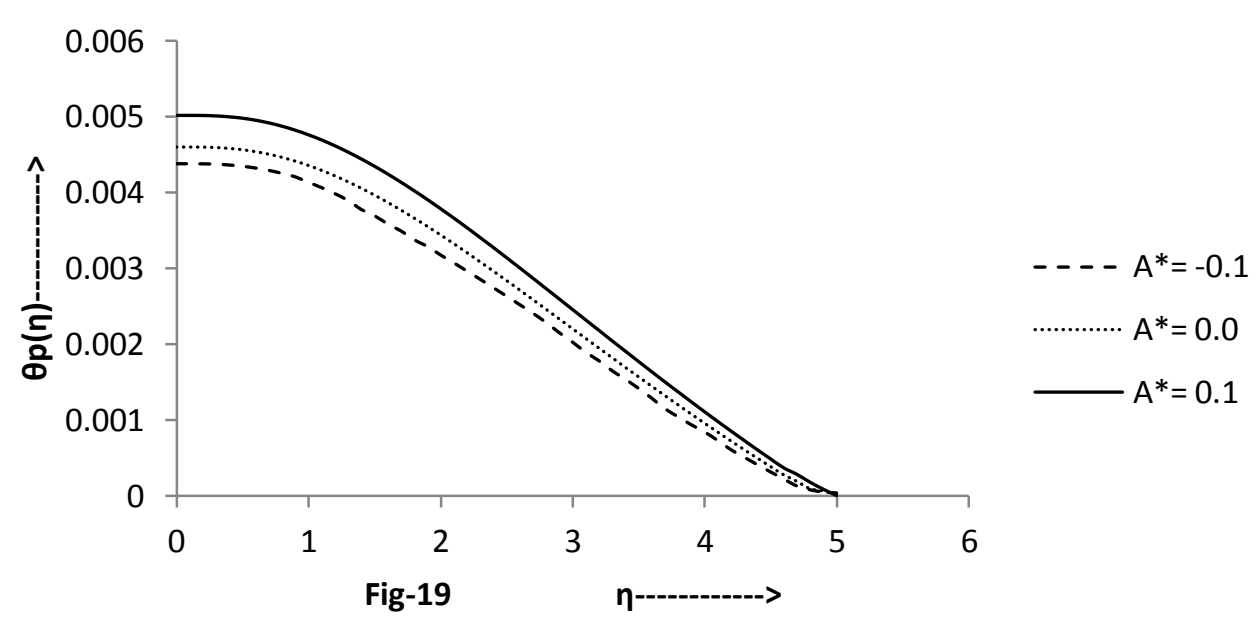

\section{Graph of $\theta$ p w.r.t.B*}

$\mathrm{EC}=1.0, \mathrm{~A}^{*}=0.1, \mathrm{Fr}=10.0, \beta=0.01, \phi=0.01$

$\operatorname{Pr}=0.71, \mathrm{Gr}=0.01, \varepsilon=3.0, \gamma=1200.0, \mathrm{~S}=5.5$

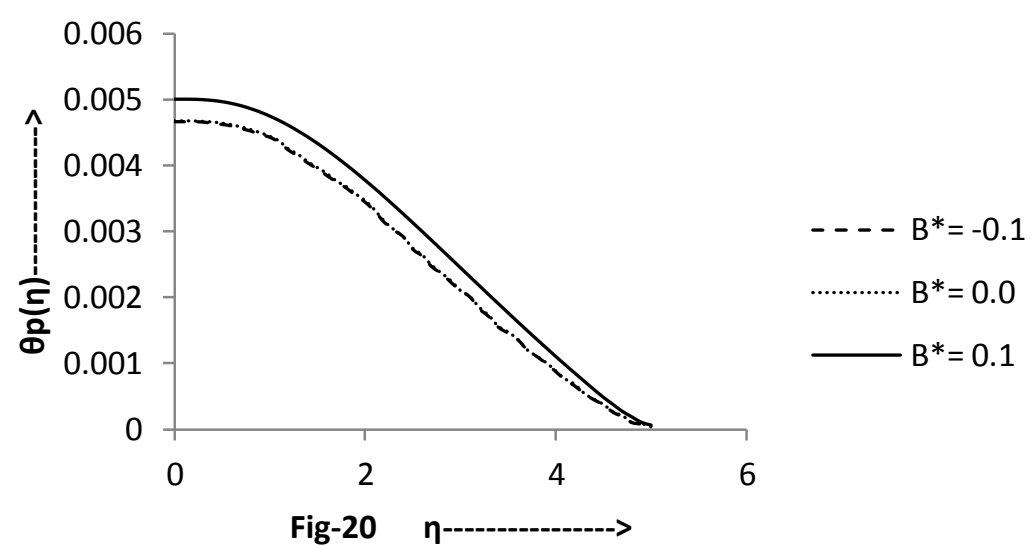




\section{RESULT AND DISCUSSION:}

The nonlinear boundary value problem described by the equations (2.6) to (2.11) subject to boundary conditions (2.12) were solved numerically, in double precision, by shooting method using the Runge-Kutta fourth order algorithm with a systematic guessing of $f^{\prime \prime}(0)$ and $\theta^{\prime}(0)$. We have investigated the problem using the values $\gamma=1200.0, \mathrm{Fr}=10.0, \epsilon=3.0$. The computations were done by the computer language FORTRAN-77.The results of heat transfer and skin friction coefficient characteristics are shown in Table-1, which shows that it is a close agreement with the existing literature. The effect of various parameters on the velocity profiles and temperature profiles also demonstrated graphically.

Fig-1 indicates the effect of Ec on velocity profile of particle phase. It is evident from the figure that increasing values of Ec increase the momentum boundary layer thickness of particle phase.

Fig-2 shows that when $\operatorname{Pr}$ is increasing, then there is significantly increasing of velocity profile of particle phase.

Fig-3 is drawn for velocity profile of particle phase which shows that, Gr has significant effect on particle phase velocity profile. It indicates that when $\mathrm{Gr}$ is increasing then the velocity distribution of dust phase increases.

Figure-4 is shown for different values of $\varphi$; it indicates that velocity profile of particle phase is increasing for increasing volume fraction.

Fig-5 witnesses the effect of $\beta$ which infers that increasing of $\beta$ increases the particle phase velocity.

Fig-6 describes that the velocity of dust phase decreases with the increasing of $S$.

Fig-7 explains the velocity profile of dust phase decreases with the increase of $A^{*}$.

Fig-8 illustrates that the velocity profile of dust phase decreases with the increase of $\mathrm{B}^{*}$.

Fig-9 shows the effect of Ec on temperature profile of fluid phase. From the figure it is evident that increasing values of Ec, the temperature of fluid phase also increases due to frictional heating of the fluid as heat energy is stored in the liquid.

Fig-10 depicts the effect of Pr on temperature profile of fluid phase. From the figure we observe that, when Pr increases the temperature of fluid phase decreases which states that the slow rate of thermal diffusion.

Fig-11 shows that for the positive value of $A^{*}$, the thermal boundary layer generate the energy, this causes the enhance of temperature of fluid phase. The negative value of $A^{*}$ indicates thermal boundary layer absorb the energy.
Fig-12 shows that the effect of $\mathrm{B}^{*}$ has the same behavior of A*.

Fig-13 illustrates the effect of Ec on temperature profile of particle phase. It is evident that the increasing of Ec decrease the temperature of dust phase which implies thermal boundary layer thickness decreases.

Fig-14 explains the effect of Pr on dust phase temperature, when $\mathrm{Pr}$ is increasing there is significantly decreasing of dust phase temperature. It means the thermal boundary layer thickness is decreasing.

Fig-15 depicts the effect of Gr on particle phase temperature profile which indicates that the increasing of Gr increase the temperature of particle phase.

Fig-16 shows the effect of $\varphi$ on temperature of particle phase. It is evident that increasing in volume fraction increases the temperature of particle phase

Fig-17 illustrates the enhancing $\beta$, increases the temperature of dust phase.

Fig-18 depicts that temperature of particle phase is increasing due to increasing of $\mathrm{S}$.

Fig-19 demonstrates that the dust phase temperature increases with increasing $A^{*}$.

Fig-20 explains about the same behavior of $\mathrm{B}^{*}$ as that of A*.

\section{CONCLUSION}

In this paper we studied the steady two phase boundary layer flow and heat transfer in a porous medium over a vertical stretching sheet including internal heat generation/absorption. The solutions of the problem have found by converting the governing partial differential equations into ordinary differential equations using similarity transformation. The effect of different parameters $\mathrm{S}, \mathrm{A}^{*}, \mathrm{~B}^{*}, \mathrm{Ec}, \mathrm{Pr}, \mathrm{Gr}, \beta, \varepsilon$ and $\varphi$ on velocity and temperature profile were examined which are interpreted graphically and discussed briefly.

It is observed from the table and figures that the following conclusions are drawn:

i. From the figures it is observed that when $\beta$ increases then velocity and temperature of dust phase increase. It also reveals that for the large value of $\beta$ i.e., the relaxation time for dust particles decrease ultimately as it tends to zero then the velocity of both fluid and dust particle will be same.

ii. Increasing value of Ec increases the temperature of fluid phase but decrease the temperature of particle phase.

iii. The thermal boundary layer thickness decreases with increase of $\mathrm{Pr}$, but increase with increasing $\mathrm{A}^{*}, \mathrm{~B}^{*}$.

iv. Increases in permeability parameter $S$ causes decrease particle velocities due to acceleration of fluid towards 
plate and particle temperature increases due to increase of $\mathrm{S}$.

v. The increasing value of $\varphi$ increases the velocity and temperature of particle phase.

vi. The values of $f^{\prime \prime}(0)$ increases with increase of Ec, $\mathrm{Gr}, \mathrm{A}^{*}, \mathrm{~B}^{*}$ and decrease with the increase of $\beta, \operatorname{Pr}, \mathrm{S}$.

vii. The values of $\theta^{\prime}(0)$ increase with increase Ec, $A^{*}, B^{*}$ ,S and decrease with the increase of $\beta, \mathrm{Gr}, \mathrm{Pr}$.

\section{NOMENCLATURE}

$q^{\prime \prime \prime}$ internal heat generation

$E_{c}$ eckert number

$F_{r}$ froud number

Department of Mathematics

S.K.C.G.College,Paralakhemundi,Odisha,India

E mail:aswinmath2003@gmail.com ,

Department of Mathematics

Centurion university of Technology and Managemant

Paralakhemundi,Odisha,India

Email:s1_mishra@yahoo.com.

$G_{r}$ grashof number

$P_{r}$ prandtl number

$T_{\infty}$ temperature at large distance from the wall.

$T_{p}$ temperature of particle phase.

$T_{w}$ wall temperature

$U_{w}(x)$ stretching sheet velocity

$c_{p}$ specific heat of fluid

$c_{S}$ specific heat of particles

$k_{s}$ thermal conductivity of particle

$u_{p}, v_{p}$ velocity component of the particle along $\mathrm{x}$-axis and $\mathrm{y}$-axis

A constant

c stretching rate

$A^{*}$ space dependent internal heat source/sink

$\mathrm{B}^{*}$ temperature dependent internal heat source/sink

$S$ permeability parameter

$\mathrm{g}$ acceleration due to gravity

$\mathrm{k}$ thermal conductivity of fluid

1 characterstic length

$\mathrm{T}$ temperature of fluid phase.

$\mathrm{u}, \mathrm{v}$ velocity component of fluid along $\mathrm{x}$-axis and $\mathrm{y}$-axis

$\mathrm{x}, \mathrm{y}$ cartesian coordinate

\section{GREEK SYMBOLS}

$\varphi$ volume fraction

$\beta$ fluid - particle interaction parameter

$\beta^{*}$ volumetric coefficient of thermal expansion

$\rho$ density of the fluid

$\rho_{p}$ density of the particle phase

$\rho_{s}$ material density

$\eta$ similarity variable

$\theta$ fluid phase temperature

$\theta_{p}$ dust phase temperature

$\mu$ dynamic viscosity of fluid

$v$ kinematic viscosity of fluid

$\gamma$ ratio of specific heat

$\tau$ relaxation time of particle phase
$\tau_{T}$ thermal relaxation time i.e. the time required by the dust particle to adjust its temperature relative to the fluid.

$\tau_{p}$ velocity relaxation time i.e. the time required by the dust particle to adjust its velocity relative to the fluid.

$\varepsilon$ diffusion parameter

$\omega$ density ratio

\section{REFERENCES}

[1] A.Adhikari and D.C.Sanyal(2013), "Heat transfer on MHD viscous flow over a stretching sheet with prescribed heat flux", Bulletin of International Mathematical Virtual Institute, ISSN 18404367,Vol.3(2013),35-47.

[2] B.J.Gireesha ,H.J.Lokesh ,G.K.Ramesh and C.S.Bagewadi(2011), "Boundary Layer flow and Heat Transfer of a Dusty fluid over a stretching vertical surface" , Applied Mathematics,2011,2,475481(http://www.SciRP.org/Journal/am) ,Scientific Research.

[3] B.J.Gireesha, A.J. , S.Manjunatha and C.S.Bagewadi(2013), " Mixed convective flow of a dusty fluid over a vertical stretching sheet with non uniform heat source/sink and radiation" ; International Journal of Numerical Methods for Heat and Fluid flow,vol.23.No.4,pp.598-612,2013.

[4] B.J.Gireesha,G.S.Roopa and C.S.Bagewadi (2013), "Boundary Layer flow of an unsteady Dusty fluid and Heat Transfer over a stretching surface with non uniform heat source/sink " , Applied Mathematics,2011 ,3,726-735 (http://www.SciRP.org/Journal/am) ,Scientific Research.

[5] C.H.Chen(1998) , "Laminar Mixed convection Adjacent to vertical continuity stretching sheet, "Heat and Mass Transfer,vol.33,no.5-6,1998,pp.471-476.

[6] Grubka L.J. and Bobba K.M(1985), "Heat Transfer characteristics of a continuous stretching surface with variable temperature" ,Int.J.Heat and Mass Transfer, vol.107,pp.248-250, 1985.

[7] H.I.Anderson,K.H.Bech and B.S.Dandapat(1992), "MHD flow of a power law fluid over a stretching sheet" , Int.J.of Nonlinear Mechanics,vol.27,no.6,pp.929-936,1992.

[8] H.Schlichting(1968), "Boundary Layer Theory", McGraw-Hill , New York,1968.

[9] Hitesh Kumar(2011), "Heat transfer over a stretching porous sheet subjected to power law heat flux in presence of heat source".Thermal Science Vol.15,suppl.2,pp.5187-5194.

[10] K .Kannan and V.Venketraman(2010), "Free convection in an infinite porous dusry medium induced by pulsating poin heat source". World Academy of Science, Engineering and Technology,63,869-877.

[11] K.M.Chakrabarti(1974), "Note on Boundary Layer in a dusty gas" ,AIAA Journal, vol.12.no.8.pp.11361137,1974

[12] L.J.Crane(1970), "Flow past a stretching plate" , Zeitschrift fur Angewandte Mathematik und physic ZAMP,VOL.2,NO.4,PP.645-647.1970. 
[13] N.Datta and S.K.Mishra (1982), "Boundary layer flow of a dusty fluid over a semi-infinite flat plate" ,Acta Mechanica,vol.42.no1-2,1982,pp7183.doi:10.10o7/BF01176514

[14] P.T.Manjunath,B.J.Gireesha and G.K.Ramesh(2014), "MHD flow of fluid-particle suspension over an impermeable surface through a porous medium with non uniform heat source/sink. TEPE,Vol.3,issue 3 august.2014,pp.258-265.

[15] R.Cortell(2005),A note on MHD flow of a power law fluid over a stretching sheet,Appl.Math.Comput.168,557-566.

[16] R.K.Ramesh , B.J.Gireesh and C.S.Bagewadi(2012), "Heat Transfer in M.H.D Dusty Boundary Layer flow of over an inclined stretching surface with non uniform heat source/sink ",Hindawi Publishing Corporation , Advances in Mathematical Physics, volume-2012,Article ID 657805,13 pages.

[17] R.N.Barik,G.C.Dash and P.K.Rath(2012), "Heat and mass transfer on MHD flow through a porous medium over a stretching surface with heat source", Mathematical Theory and Modeling, ISSN 22245804,Vol.2,No.7,2012.

[18] Robert A.Van Gorder and K.Vajraveluet.al(2011), "Convective heat transfer in a conducting fluid over a permeable stretching surface with suction and internal heat generation/absorption. Applied Mathematics and computation, 217,5810-5821.

[19] Saffman ,P.G.(1962), "On the Stability of Laminar flow of a dusty gas", Journal of Fluid Mechanics, 13,120-128.

[20] Sakiadis B.C(1961), "Boundary Layer behavior on continuous solid surface ; boundary layer equation for two dimensional and axisymmetric flow" A.I.Ch.E.J,Vol.7,pp 26-28.

[21] Sharidan S. , Mahmood J. and Pop I. , "Similarity solutions for the unsteady boundary layer flow and Heat Transfer due to a stretching sheet", Int.J.of Appl.Mechanics and Engeenering,vol.11,No.3,pp 647-654.

[22] Singh, P.K.,Singh,J.(2012), "MHD flow with viscous dissipation and chemical reaction over a stretching porous plate in porous medium". Int.J. of Engneering Research and Applications.Vol.2,No.2,pp.1556-1564.

[23] Subhas , A.M. and N.Mahesh(2008), "Heat transfer in MHD visco-elastic fluid flow over a stretching sheet with variable thermal conductivity, non-uniform heat source and radiation ,Applied Mathematical Modeling,32,1965-83.

[24] Swami Mukhopadhayay(2012), "Heat transfer analysis of unsteady flow of Maxwell fluid over stretching sheet in presence of heat source/sink.CHIN.PHYS.LETT.Vol.29, No.5054703.

[25] Vajravelu , K. and Nayfeh (1992), J. , "Hydromagnetic flow of a dusty fluid over a stretching sheet" , Int.J. of nonlinear Mechanics,vol.27,No.6,pp.937-945.

\section{BIOGRAPHIES}

Aswin Kumar Rauta was born in Khallingi of district Ganjam, Odisha, India in 1981.He obtained the M.Sc. and M.Phil. degree in Mathematics from Berhampur university, Berhampur, Odisha, India. He joined as a lecturer in Mathematics in the Department of Mathematics, S.K.C.G.College, Paralakhemundi ,Odisha,India in 2011 and is continuing his research work since 2009 and work till now. His field of interest covers the areas of application of boundary layer,heat/mass transefer and dusty fluid flows.

Dr.Saroj Kumar Mishra was born in Narsinghpur of Cuttuck district,Odisha,India on $30^{\text {th }}$ june 1952. He received his M.Sc. degree in Mathematics (1976) and Ph.D in Mathematics in 1982 on the research topic "Dynamics of two phase flow" from IIT Kharagpur, India . Currently he is working as Adjunct Professor of Mathematics at Centre for Fluid Dynamics Research, CUTM, Paralakhemundi, Odisha, India. He has authored and coauthored 50 research papers published in national and international journal of repute. He has completed one Major Research project and one Minor Research project sponsored by UGC, New Delhi, India. He has attended/presented the papers in national, international conferences. He is a member of several bodies like Indian Science Congress Association, Indian Mathematical Society, ISTAM, OMS, and BHUMS etc. His research interest includes the area of fluid dynamics, dynamics of dusty fluid particularly, in boundary layer flows, heat transfer, MHD, FHD and flow through porous media. His research interest also covers the nano fluid problems, existence and stability of problems and other related matters. Eight students have already awarded Ph.D degree under his guidance and another six students are working under his supervision. 
Table-1 Showing initial values of wall velocity gradient $-f^{\prime \prime}(0)$ and temperature gradient $-\theta^{\prime}(0)$

\begin{tabular}{|c|c|c|c|c|c|c|c|c|c|c|c|c|c|}
\hline$A^{*}$ & B* & $\mathbf{S}$ & $\boldsymbol{\beta}$ & $\boldsymbol{P}_{r}$ & $E_{c}$ & $\varphi$ & $G_{r}$ & $-f^{\prime \prime}(0)$ & $u_{p}(0)$ & $-v_{p}(0)$ & $\mathbf{H}(\mathbf{0})$ & $-\boldsymbol{\theta}^{\prime}(\mathbf{0})$ & $\boldsymbol{\theta}_{p}(\mathbf{0})$ \\
\hline & & & - & 0.71 & 0.0 & 0.0 & 0.0 & 1.001397 & - & - & - & 1.082315 & - \\
\hline \multirow[t]{18}{*}{0.1} & 0.1 & 5.5 & 0.01 & 0.71 & 1.0 & 0.01 & 0.01 & 1.083264 & 0.005211 & 0.990469 & 0.198966 & 0.641181 & .0050144 \\
\hline & & & & & 2.0 & & & 1.082108 & 0.005497 & 0.990776 & 0.198602 & 0.357561 & 0.002625 \\
\hline & & & & & 3.0 & & & 1.080386 & 0.005644 & 0.990994 & 0.200646 & 0.076176 & 0.002915 \\
\hline & & & & 0.5 & & & & 1.082725 & 0.005261 & 0.989691 & 0.207921 & 0.481854 & 0.005065 \\
\hline & & & & 0.71 & & & & 1.083264 & 0.005211 & 0.990469 & 0.198966 & 0.641181 & .0050144 \\
\hline & & & & 1.0 & & & & 1.083207 & 0.005435 & 0.990755 & 0.199126 & 0.826194 & 0.003271 \\
\hline & & & & & & & 0.01 & 1.083264 & 0.005211 & 0.990469 & 0.198966 & 0.641181 & .0050144 \\
\hline & & & & & & & 0.02 & 1.076393 & 0.005677 & 0.990981 & 0.200328 & 0.654672 & 0.005207 \\
\hline & & & & & & & 0.03 & 1.070957 & 0.005703 & 0.991110 & 0.198726 & 0.655146 & 0.004827 \\
\hline & & & & & & 0.01 & & 1.083264 & 0.005211 & 0.990469 & 0.198966 & 0.641181 & .0050144 \\
\hline & & & & & & 0.02 & & 1.082553 & 0.005466 & 0.990789 & 0.199096 & 0.646527 & 0.005129 \\
\hline & & & & & & 0.03 & & 1.082562 & 0.005487 & 0.990797 & 0.199106 & 0.646529 & 0.005135 \\
\hline & & & 0.01 & & & & & 1.083264 & 0.005211 & 0.990469 & 0.198966 & 0.641181 & .0050144 \\
\hline & & & 0.02 & & & & & 1.083440 & 0.010901 & 0.981992 & 0.198161 & 0.646130 & 0.010128 \\
\hline & & & 0.03 & & & & & 1.085183 & 0.015188 & 0.971952 & 0.197745 & 0.642598 & 0.016976 \\
\hline & & 5.0 & & & & & & 1.091158 & 0.005551 & 0.990396 & 0.198733 & 0.638913 & 0.004755 \\
\hline & & 5.5 & & & & & & 1.083264 & 0.005211 & 0.990469 & 0.198966 & 0.641181 & .0050144 \\
\hline & & 6.0 & & & & & & 1.075638 & 0.005600 & 0.990853 & 0.199686 & 0.649527 & 0.005038 \\
\hline-0.1 & & & & & & & & 1.082903 & 0.005446 & 0.990768 & 0.199107 & 0.765160 & 0.004382 \\
\hline 0.0 & & & & & & & & 1.083768 & 0.005418 & 0.990504 & 0.198925 & 0.700226 & 0.004596 \\
\hline 0.1 & & & & & & & & 1.083264 & 0.005211 & 0.990469 & 0.198966 & 0.641181 & .0050144 \\
\hline & -0.1 & & & & & & & 1.083481 & 0.005413 & 0.989971 & 0.191079 & 0.792324 & 0.004664 \\
\hline & 0.0 & & & & & & & 1.084334 & 0.005380 & 0.990063 & 0.207911 & 0.722986 & 0.004675 \\
\hline & 0.1 & & & & & & & 1.083264 & 0.005211 & 0.990469 & 0.198966 & 0.641181 & .0050144 \\
\hline
\end{tabular}

